\title{
A Theoretical Study to Realize Solitonic 2: 1 Multiplexer Using Coupling Actions of Optical Pulse
}

\author{
P. Kuila* \\ Dept. of Physics, Midnapore College (Autonomous), Vidyasagar University, Midnapore, India \\ *Corresponding author : puspendu77@rediffmail.com mob:09734666104
}

Available online at: www.isroset.org

Received: 21/Nov/2018, Accepted: 12/Dec/2018, Online: 31/Dec/2018

\begin{abstract}
In combinational logic system, multiplexer is an important part where one can select a particular input in the output side. The specific optical input to be selected at the exit side of the scheme is determined by the control or select channel. Here, 2:1 optical multiplexer is proposed by using the coupling action of the optical pulse of a biased optical coupler. The electro-optic nature of the optical wave guide are massively used to convert the optical pulse into optical soliton pulse. Optical soliton pulses are used here as a substitute of ordinary optical pulse as the information carrier due to some superior character of the soliton pulse. Before introducing in the coupling region. Optical pulse should be converted into solitonic one. Such category of multiplex system may be used for ultra-high speed remote operation and for high secure communication system.
\end{abstract}

Keywords- Optical communication, Coupled waveguide, Evanescent optical pulse, EO effect, Optical soliton pulse.

\section{INTRODUCTION}

Multiplexer is a combinational logic system that has maximum of $2^{\mathrm{n}}$ data inputs, $\mathrm{n}$ controlled channels and only one output line. One of these data inputs will be transferred to the output based on the values of selection lines.

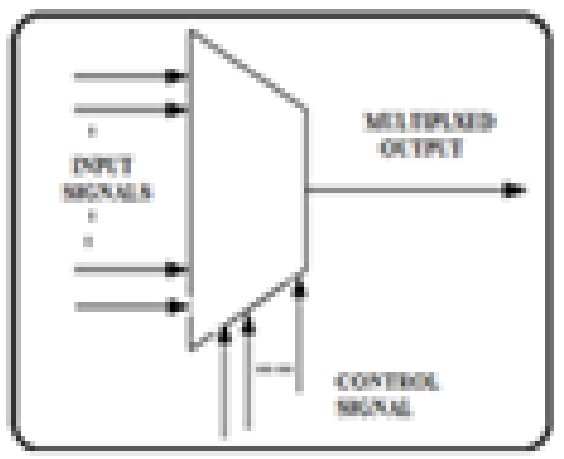

Figure 1: Symbol of multiplexer

A multiplexer are used to select one input signal to the output according to the control signal. Due to this function, a multiplexer is also called a data selector. For long distance operation such data selector scheme is very important. The schematic symbol for a multiplexer is as shown in fig: 1 .

In a 2:1 mux, there are only two inputs and to control the inputs to the outlet side only one controlled line is sufficient. The schematic symbol for a 2:1 multiplexer is as shown in fig: 2 .

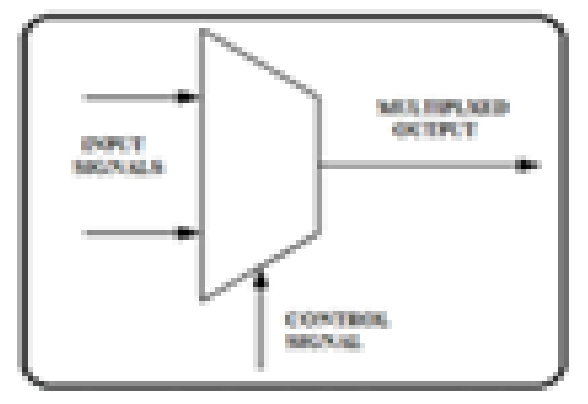

Figure 2: Symbol of 2:1 multiplexer

In evanescent wave coupling optical pulses are transmitted from one optical wave guide to another. If the separation between the two wave guides becomes so small that the evanescent waves of the two wave guides overlap to a considerable extent, then there is a conversation of energy between the two wave guides. In this situation, the evanescent field associated with the propagating modes in the two wave guides interact and lead to a periodic exchange of energy between the two wave guides. Such type of wave guides where coupling between two guides follows is designated as directional coupler. There is a wide-ranging transfer of energy from one to other wave guide will occurs if the propagation constant of the modes in the two wave guides are matching and else there is a partial handover of energy $[1,2,3]$. Again when an optical pulse having a well 
defined power shape propagates through an optical fiber, at which the dispersion effect and non-linear effect exactly compensate to each other then a stable wave packet is made. These distinct types of pulses are considered as optical soliton pulse (OSP) which does not broaden in time domain or in frequency domain when it travels through the nonlinear optical fiber medium. Due to such inherent character of the OSP, it is better to use as information carrier in place of ordinary optical pulse $[4,5]$.

In this communication, implementation of optical soliton based 2:1 mux is proposed by using the optical coupling nature of the pulse in the coupling region of the directional coupler. Such theoretical approach is suitable for very high speed and highly secure remote operation.

\section{METHODS AND MATERIALS}

If $\mathrm{A}_{2}(\mathrm{z})$ represent the amplitude of the evanescent wave in the $2^{\text {nd }}$ wave guide due to optical pulse of amplitude $A_{1}(z)$ incident in $1^{\text {st }}$ wave guide in a directional coupler, ( as shown in fig. 3) then the optical power in $1^{\text {st }}$ and 2 nd waveguide after interaction become respectively as proportional to $\left|A_{1}(z)\right|^{2}$ and $\left|A_{2}(z)\right|^{2}$ as given in equ. (1) and (2) respectively. where

$\left|A_{1}(z)\right|^{2}=1-\frac{k^{2}}{\theta^{2}} \operatorname{Sin}^{2}(\theta z)$

$\left|A_{2}(z)\right|^{2}=k^{2} \frac{\operatorname{Cos}^{2} \theta z}{\theta^{2}}$,

$\mathrm{k}=\left(k_{12} k_{21}\right)^{1 / 2} \quad$ [ where, $k_{12}, k_{21}$ are the strength of interaction between two modes],

$\theta=\left[\frac{1}{4}(\Delta \beta)^{2}+k^{2}\right]^{1 / 2} \quad$ [where, $\Delta \beta=\beta_{1}-\beta_{2}, \beta_{1}$ and $\beta_{2}$ are the propagation constants of the mode in the wave guide-1 and wave guide- 2 respectively] [6]. Equations (1) and (2) shows the periodic exchange of energy between two couplers whose frequency of exchange of energy depends not only the properties of the couplers materials but also on the dimension of the coupling region. Again maximum energy will be exchanged from one to other wave guide will be happen for a minimum coupling length between two coupler is given as

$$
Z_{c}=\frac{\pi}{2 \sqrt{(\Delta \beta / 2)^{2}+k^{2}}}
$$

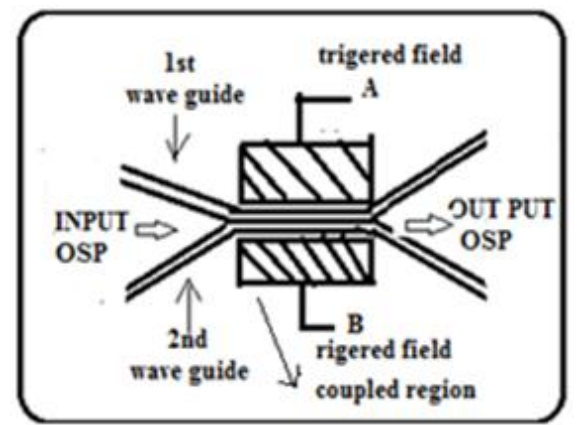

Figure 3: Typical Biased directional coupler If $\beta_{1}$ and $\beta_{2}$ are perfectly same for two guiding medium, then the coupling length becomes as

$Z_{\text {co }}=\frac{\pi}{2 k}$

Now, if we place two wave guide such that the coupling length become exactly $Z_{c o}$, then optical pulse will be switched to the other in the coupling region, this case is denoted as cross state. If some how we make $\Delta \beta=2 \sqrt{3} k$ then the new coupling length will be as $1 / 2 Z_{c o}$. In the later case, the energy exchanged will be stopped and optical pulse will be persist in the same guide, this is designated as parallel state.

The electro-optic behavior of the optical wave guides are used here to switch over from cross state to parallel state. Electric field are applied in the coupling region in opposite sense w.r.t each guide, so that, effective refractive index of one guide increases where as for other guide it is decreases (as shown in fig:3). The electric field is applied exactly along the c-axis as the incident light is along the c-axis. Before incident of the optical pulse into the guides, the optical pulses are changed to a precise power profile so that the optical pulse transferred to optical soliton pulse. Already we proposed some very effective process to get OSP from ordinary optical pulse. [6 ].

\section{RESULT AND DISCUSSION}

Fig. (4) shows the schematic block of the proposed optical multiplexer system. Here two directional coupler are used back to back situation.

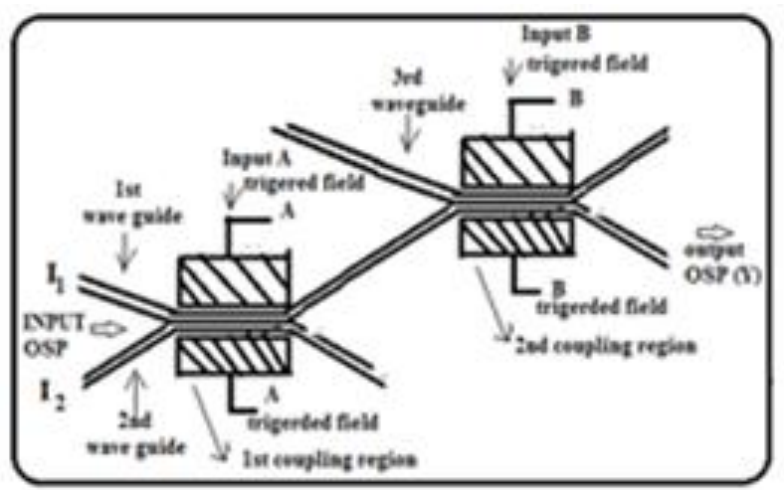


Figure 4: Schematic diagram of the proposed optical multiplexer

The 1 st coupler is intended by coupling $1^{\text {st }}$ and $2^{\text {nd }}$ wave guide, where as the second coupler is shaped by pairing the outlet of $1^{\text {st }}$ wave guide from first coupler and an another similar $3^{\text {rd }}$ wave guide. Oppositely focused electric field having a pre-specified values are applied in both the couplers in coupling section, such that the situation $\Delta \beta=$ $2 \sqrt{3} k$ have been fulfilled in case of each couplers.

$I_{1}, I_{2}$ symbolize the solitonic optical inputs at the input face of the $1^{\text {st }}$ wave guide and $2^{\text {nd }}$ wave guide respectively. Before introducing the optical pulse into the waveguide, it is converted into solitonic pulse. The state (present or absent) of the external electric field applied at the coupling region are considered as inputs of the proposed scheme. The state of the triggering field applied to the $1^{\text {st }}$ coupler is considered as input $\mathrm{A}$ and that for $2^{\text {nd }}$ coupler is considered as input $\mathrm{B}$. The input state is considered as state zero (0) only when the triggered field is applied. In this situation the coupler is in parallel state so that almost all the signal remain in the same wave guide i.e. coupling effect becomes very negligible. The input state is considered as state one when the external triggering field is absent. Here the coupler will be in cross state, where almost all the optical signal transfer to another waveguide in the coupling region and the coupling effect will be maximum. The optical soliton signal (Y) emerges from $1^{\text {st }}$ wave guide after the $2^{\text {nd }}$ coupling region is considered as out put of the proposed multiplexer. The out put is considered as state one only when a considerable high intense OSP is come out from the optical wave guide. At the same time the output state is considered as state zero when a very low intense or zero intense optical pulse is obtained at the outlet of the $1^{\text {st }}$ wave guide at $Y$.

To implement the proposed scheme, OSP are incident on both the $1^{\text {st }}$ and $2^{\text {nd }}$ waveguide in $1^{\text {st }}$ coupler i.e. both $I_{1} \&$ $I_{2}$ are present. Also the triggering field is remain applied through out the operation. Here, Input A behaves like control line and $\mathrm{Y}$ is the multiplexed output for the proposed 2:1 MUX.

Case 1: When $A=0$ : In such case, biasing field in both the coupler are present and both the coupler remain in parallel state. Hence the optical signal $\left(I_{1}\right)$ in the $1^{\text {st }}$ guide remains in the same guide as the coupling effect are nullified in both the coupler in this case. Therefore, $I_{1}$ signal will be transferred at the outlet side.

Case 2: When $A=1$ : In this case, $1^{\text {st }}$ coupler will be in cross state where as $2^{\text {nd }}$ coupler remains in parallel state. Hence, optical pulse $I_{2}$ will be obtained at Y.

Therefore, one can select the output by controlling the triggering signal in the $1^{\text {st }}$ coupling region only. The situation is represent in a tabular form in table 1 .
Table 1: Different state of input and output

\begin{tabular}{|c|c|c|}
\hline Situation & $\begin{array}{c}\text { A } \\
\text { (control line) }\end{array}$ & $\begin{array}{c}\text { Y (multiplexed } \\
\text { output) }\end{array}$ \\
\hline Case 1 & 0 & $I_{1}$ \\
\hline Case 2 & 1 & $I_{2}$ \\
\hline
\end{tabular}

\section{CONCLUSION}

Here, the coupling nature of the wave guide and EO effect of the guiding medium are both used extensively. EO effect are used mainly to convert the optical signal to solitonic one. Hence the proposed system will be very much effective for the high distance and high secured operation. The polarization state of the input optical soliton pulse should be maintained in same manner for proper operation. One can also reduce the value of required triggering pulse by suitably alter the electrode dimension. It is very important to note that the coupling length should be adjusted according to the non-linearity of the guiding medium as the intensity of the soliton pulse is high.

\section{ACKNOWLEDGEMENT}

This paper is a portion of Minor Research Project sponsored by UGC and the author is thankful to the UGC for providing the financial assistance to carry out the project.

\section{REFERENCES}

[1] A. Ghatak and K. Thyagarajan. Optical Electronics. (Cambridge University Press, New Delhi, 2006).

[2] A.Hsegawa. Optical solitons in fibers. ( Springer verlag . Newyork, 1989).

[3] G.P.Agarwal, Fiber optic communication system, John Wiley. Singapore, (1993).

[4] A. Sinha and S. Mukhopadhyay, "Effect of higher order nonlinearity in frequency variation of self phase modulationin optical fiber communication", Chinese Opt. Letts., 2(9), 500-502(2004).

[5] P.Kuila, A.Sinha, H. Bhoumik and S. Mukhopadhyay , "A theoretical study of using amplitude modulation scheme of an electro optic modulator for generation of proper power shape function of an optical soliton pulse in a non-linear wave guide", Optical Engineering (SPIE, USA), 45(4), 045002, 2006.

[6] P. Kuila, "An analytical study to realize soliton based optical AND logic operation using coupling behavior of optical coupler", IJSRCSEIT, (3)1, Jan-Feb 2018, 522-526.

\section{AUTHORS PROFILE:}

Dr. Puspendu Kuila obtained his M.Sc degree in 1999 from Vidyasagar University in West Bengal. He was a gold medalist at Vidyasagar University in West Bengal for his first position in the graduate degree program there. He has obtained his Ph.D. degree in 2011 from Vidyasagar University in West Bengal. His research field is optical soliton communication system. In this area he already published eleven publications in reputed international journals and presented more than twenty five presentation in different national and international seminar in India. Now, he is working as an Assistant Professor in Physics in Midnapore College (Autonomous), West Bengal. He has more than 16 years of teaching experience and 11 years of Research Experience. 